\title{
Trends in serious head injuries among cyclists in England: analysis of routinely collected data
}

\author{
Adrian Cook, Aziz Sheikh
}

As the health and environmental benefits of cycling have become better appreciated, successive governments in the United Kingdom have encouraged cycle use. Cyclists, however, face considerable risk of injury, of which head injuries most commonly result in serious adverse outcomes. Despite evidence from casecontrol and time trend studies, ${ }^{12}$ questions remain about the effectiveness of helmets, particularly for adults. We examined trends in emergency admissions for cycle injuries to English hospitals between 1991 and 1995, during which time the wearing of helmets increased (Research International Ltd, personal communication). ${ }^{3}$ into the hospital episode statistics database. We studied the data for the period 1 April 1991 to 31 March 1995, at which time diagnoses were classified according to ICD-9 (international classification of diseases, ninth revision), and extracted all records concerning cyclists, whether their injuries resulted from bicycle accidents or motor vehicle accidents (codes E8261, E810-E825 fourth digit $=6$ ). From information in the primary diagnosis field, we identified head injuries as either "fracture of vault or base of skull" (ICD-9 800, 801) or "intracranial injury" (ICD-9 850-4). We used only data concerning emergency admissions and completed first episodes.

We used monthly counts to calculate the number of cyclists admitted with head injuries as a percentage of the total number of cyclists admitted and divided the patients into three age categories: junior (6-10 years), secondary (11-15 years), and adult (16 years and over). Using the percentage of head injuries per month as the outcome variable, we assessed trends over time for significance by fitting four linear regression models-one to the complete dataset (figure) and one to each of the three age categories.

Of the 12.6 million emergency admissions in the study period, $35056(2.8 \%)$ were for injuries sustained while cycling. The average length of stay was 3.3 days. 'Head injuries' was the primary diagnosis in 34\% $(n=11985)$ of these admissions, over half of which $(\mathrm{n}=7531)$ were among children aged $<16$ years. One per cent of cyclists $(n=121)$ admitted with head injuries died as a result of their injuries.

Numbers of emergency admissions among cyclists changed little over the four years of the study period: from 8678 in 1991-2 to 8781 in 1994-5. However, the number with head injuries as the primary diagnosis fell from 3393 to 2571 . The regression models showed a $12 \%$ reduction $(95 \%$ confidence interval $10 \%$ to $15 \%$ )-from $40 \%$ to $28 \%$-in the number of cyclists admitted with head injuries as a percentage of total monthly admissions. A reduction occurred in each age group during the study period. As a percentage of total earlier drafts of this paper.

(Accepted 17 July 2000)

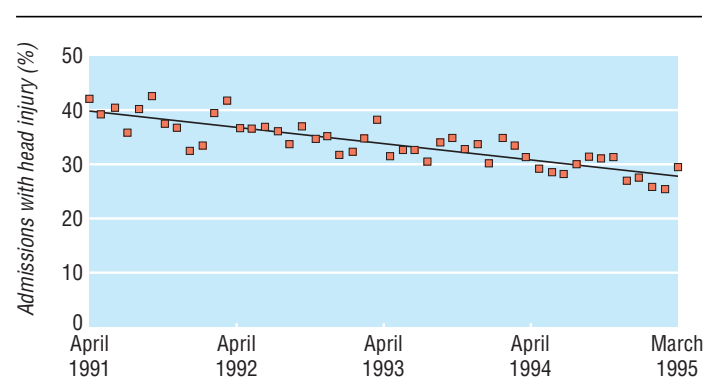

Linear regression of head injury admissions as a percentage of total admissions among cyclists, 1991-5

admissions the reductions were estimated as: junior, $9 \%(95 \%$ confidence interval $3 \%$ to $16 \%)$; secondary, $11 \%(7 \%$ to $16 \%)$; and adult, $13 \%$ (11\% to $16 \%)$.

\section{Department of Primary Health Care and General Practice, Imperial College School of Medicine, London W2 1PG \\ Adrian Cook statistician \\ Aziz Sheikh NHS REDD training fellow \\ Correspondence to: A Cook a.d.cook@ic.ac.uk}

BMJ 2000;321:1055

\section{Comment}

The number of serious head injuries among cyclists fell markedly during a period of increasing helmet use, suggesting that helmets offer protection. Case-control studies have shown the benefit of wearing helmets, ${ }^{1}$ though inadequate control for possible confounding factors means that this conclusion is uncertain. ${ }^{4}$ Analysis of injury trends in Australia has also shown benefits, ${ }^{2}$ but these results were affected by a reduction in bicycle use when helmet wearing became compulsory. We accounted for any change in cycle use by using the total number of cyclists admitted as the denominator.

Our findings indicate that cycle helmets are of benefit both to children and, contrary to popular belief, to adults. The reason that people most frequently cite for not cycling is risk of injury; measures to increase cycle use must therefore address safety. Local publicity campaigns encouraging the voluntary wearing of helmets have been effective and should accompany national drives to promote cycling.

We thank Debbie Hart for her assistance in conducting literature searches and Liam Smeeth, Brian Hurwitz, Paul Aylin, Sasha Shepperd, and Bernadette Alves for their comments on

Contributors: AC conceived the research idea. AC and AS jointly devised the study protocol and wrote the paper. AC analysed the data and is the guarantor for the study.

Funding: AS is in receipt of an NHS R\&D National Primary Care training award.

Competing interests: None declared.

1 Thomspon RS, Rivara FP, Thompson DC. A case-control study of the effectiveness of bicycle safety helmets. N Engl J Med 1989;320:1361-7.

2 Cameron MH, Vulcan AP, Finch CF, Newstead SV. Mandatory bicycle helmet use following a decade of helmet promotion in Victoria, Australia - an evaluation. Accid Anal Prev 1994:26:325-7.

3 Wardle S, Iqbal Z. Cycle helmet ownership and wearing; results of a surWardle S, Iqbal Z. Cycle helmet ownership and wearing; result
vey in South Staffordshire. J Public Health Med 1998;20:70-7.

4 McCarthy M. Do cycle helmets prevent serious head injury? BMJ 1992;305:881-2. 